During the past year-1860-six cases have come under my care which were illustrative of this practical poirit. They show also that epipbora of apparently congenital origin is due to other causes than insufficient development of the osseous parietes of the tear-canal, or hereditary predisposition.

The subjects of the disease were healthy, but diathetically strumous. In one, an infant of eight months, in whom there were grounds for suspecting a syphilitic taint, acute abscess of the sac threatened; it was swollen, tense, and red. The canaliculus was slit while the child was under chloroform, and a probe was passed into the nasal duct, with the effect of removing the urgent symptoms in thirty-six hours. The five other cases were treated in the same way, with the addition of astringents. The attendants should be directed to press out the saccontents every two or three hours.

The duty of examining the condition of the tear-sac in all cases of infantile or adult purulent ophthalmia will not need, after what has been said, to be urged upon surgeons.

\section{ON SORE-THROAT.}

By Thomas Inmax, M.D.Lond., Physician to the Liverpool Royal Infirmary.

THE subject of sore-throat is so familiar, that few think it now-a-days worth much consideration.

that it is so, the following observation will prove.

If we ask ourselves what we mean by the words sorethroat, the natural response is, " a throat which is sore" -but not content with this, we add, that the throat is sore because it is affected by inflammation or by ulceration; and we still farther localise our ideas, by saying that the affection is one of the tonsils or mucous membrane; so that the ultimate answer to the question is "the throat is sore, because the mucous membrane is inflamed or ulcerated;" and as a result, all our remedial measures are directed to curing this abnormal condition, by astringent or stimulating gargles, the use of caustic, and the like.

But if we prosecute our investigation a little farther, we shall find that we have been contented with superficial information. "Why," we ask, " is inflammation and ulceration of the mucous membrane attended with soreness?" and "why do we have soreness, as in some forms of dysphagia, when such mucous affections are absent?" Again, we sometimes see the throat inflamed but not sore; and we most of us know practically, that when ulceration exists extensively, little pain is complained of except during the act of swallowing ; this leads us to the idea, that the "soreness" is chiefly connected with, and dependent upon deglutition.

The only exception to this is in that condition of the throat to which the name of relaxation is given, in which we find that the act of sivallowing positively gives relief. This I shall explain by and bye.

Having arrived thus far we have to inquire "why is deglutition so painful in cynanche?" The answer is readily given. 'The act is a muscular one, involving the associate contraction of the fancial and pharyngeal muscles, which are generally implicated by an extension of inflammation from the mucous membranes. But is it a fact, that muscles in contact with inflamed surfaces are commonly and painfully affected? Unquestionably; for we all know how severe is the affection of the intercostals in herpes zoster or in pleurisy, and of the abdo. minal muscles in cases of peritonitis. We know how acutely painful is muscular contraction near a goutily inflamed joint, or in acute rheumatism.

The idea being once started, that the pain in cynanche is myalgic, we next inquire whether it may not be pro. duced by causes external to the pharynx; we turn to experience, and find that sore throat attends inflamma- tion of the parotid. I have now repeatedly seen it attend $C$ myositis of the sterno-mastoid, in which case the spread $\Rightarrow$ of inflammation from this muscle to the pharynx, and $\stackrel{\vec{S}}{\rightarrow}$ ultimately to the mucous membrane of the throat, has $\bar{\sigma}$ been well marked.

We have dysphagia accompanying myalgia of the $\frac{\bar{O}}{\bar{S}}$ sterno-mastoid, sterno-thyroid, and sterno-hyoid muscles. $\frac{\text { D }}{\partial}$ We have sore throat from prolonged shouting, or speaking in a high key; and I have known a similar pheno-
menon from severe paroxysmal cough.

The pain of relaxed sore-throat is readily explained $\overrightarrow{\overrightarrow{0}}$ on the muscular hypothesis-for it clearly resembles? that which is felt when the cremaster, being flabby and $\overrightarrow{\vec{c}}$ soft, is stretched to its utmost extent by the weight of $\vec{\omega}$ the testicle. It arises from the constant drag on the levator and tensor palati, and is also temporarily relieved by pressing the soft palate firmly upwards with the finger or any hard substance. The contraction of these $-\vec{A}$ muscles, which occurs during deglutition, also renders their fibres more tense for a short period, and thus gives $\infty$ relief from the tiresome dragging pain.

The hypothesis I thus advance is not barren of $\frac{0}{2}$ practical results. If it be true that the chief sufferings $N$ in cynanche are due to the forced contraction of inflamed or irritated muscles, it follows that any irritating appli- ֻे cations are likely to increase the pain. Do they act thus in reality? Certainly - we have seen a child with diph- ㄱ theria, unable to swallow wine in any form, and even a weak solution of tincture of iron, without spasmodically $\vec{\infty}$ rejecting a quantity through the nostrils, and almost screaming with pain, which was, from the fingers being.driven into the ears in that direction, evidently referred $\square$ to the attachment of the lingual, faucial, and pharyngeal muscles to the styloid process. Yet, this patient could readily swallow milk and other bland fluids without any $\bar{O}$ suffering or muscular spasm. The application of nitrate of silver never gave her relief-she said it made her worse. Another patient, an adult lady, with scarlatina 2 ulceration of the throat, presented a similar phenomenon. $\overrightarrow{\vec{B}}$ She was equally unable to take wine without agonising pain-yet she could swallow barley water with ease and comfort, and all local applications of caustic solutions made her worse. The theory farther points to a new? principal of local treatment, viz.-we must try to relieve the irritability of the muscles, as well as to heal the mucous membrane. Acting on this idea, I have used opiate epithems externally for some time, and a few weeks ago began to use laudanum and water as a gargle. The effects have surpassed my expectations, and no case could more fully corroborate my views, than the follow-윽 ing. Mr. C. aged 30, after being much exposed to scarlatina, had ulcerated sore throat: this was so ex-o quisitely painful, that he was unwilling to swallow anything; and he could not sleep, for he was constantly awaked by involuntary efforts of deglutition, which $D$ occurred as soon as he began to be oblivious; and he could not even speak intelligibly. I used no internal application whatever but a gargle of laudanum and water. N He reported the effect as magical, for he was able both to N speak and swallow comfortably as soon as he had finished gargling; the ulceration remained, the soreness had gone $\mathrm{He}$ had only to repeat the application three times in all The only adjunct I adopted was the use of iodine paint $\frac{\mathbb{D}}{\mathbb{D}}$ externally.

The effect of anodyne gargles is not merely to diminish? the irritability of the muscles and the constant desire to swallow, which is so provoking an accompaniment of cynanche; they have also a sedative influence on the mucous membrane, and diminish the amount of its se- $\frac{?}{9}$ cretion, the abundance of which is so distressing in many cases; and in one remarkable case which came under myo care, many years ago, in which there was general stomatitis as well as inflammation of the fauces and pharynx, total inability to $\mathrm{swallow}$ anything or to articulate, witho a perpetual discharge of very dense mucus, the use of 
laudanum and water as a wash was the first and only thing which gave relief.

We may sum up our views shortly thus. The pain of sore-throat and dysphagia is due to the muscles of deglutition being unusually irritable or inflamed, and in the treatment of these affections regard must be had to these muscles, as well as to the condition of the mucous membrane covering them. The new plan of treatment does not materially militate against the old, and the two may always be combined in one way or another.

I may add in conclusion, that the anodyne treatment is applicable to all forms of cynanche-whether it is catarrhal, scarlatinal, diphtheritic, syphilitic or croupy; and that I have not yet found the laudanum act prejudicially in any case.

While on the subject let me add, that before I adopted this plan I was in the habit of prescribing a small piece of camphor to be constantly retained in the mouth in cases of cynanche, tonsillitis, and for sore-throats generally. In the former I know nothing equal to it-in the latter it is inferior to opium.

\section{Trambactions of 迡ramethes.}

\section{EAST YORK AND NORTH LINCOLN BRANCH.}

ON DIPHTHERIA AND ITS ASSOCIATED DISEASES, AS THEY APPEARED AT BARTON-UPON-HUMBER AND THE NEIGHBOURHOOD IN THE YEARS 1858 AND 1859.

By W. H. Edpre, Esq., President of the Branch.

[Read September 26th, 1560.]

THE epidemic of diphtheria which visited Barton and the neighbourhood during 1858 and 1859, exhibited many important facts which cannot fail to be interesting to the members of the Association, not only as regards the disease, pure and simple, but also its associated diseases and sequelie.

The town is chietly built on a substratum of shingly gravel and sand, under the edge of the chalk formation, in which there is here a break for the passage of the estuary of the Humber. From this latter, the town is distant about a mile, with a gentle declivity, affording an excellent opportunity for drainage. This has been taken advantage of to some extent, the principal part of the streets having been tunnelled; there is, however, much yet to be done before the town can be considered complete in a sanitary point of view. From the situation of the town, so wear a large tidal river, the air is generally pure, but cold in the spring, from its exposure to the east, from which quarter the wind usually blows at that time of year.

Scarlatina, which had not been epidemic amongst us for many years, had been prevalent in the neighbouring villages to the west of Barton during the spring and summer of 1858, but was of a mild character in num. bers of cases, not requiring any medical treatment; and it was in June of that year that I saw the first cases of a malignant character, at the village of Saxby, five miles from Barton. There were three children in a family of four, of the respective ages of 5,3 , and 2 years, and a younger one, 14 months old. The eldest was the first attacked, and died in four days of malignant scarlatina. The third child, a boy, aged 2 years, fell ill the day before the first died; he was carried off by effusion on the brain before the eruption had fully come out, or any serious affection of the throat made its appearance. The second now began to be ill, but had the scarlatina much less severely than the other two; the throataffection being but slight; and, although she had ana. sarca, with albuminuria, she ultimately recovered. The youngest child was the last attacked; and in him the scarlatina was followed by diphtheria. As the former disease declined, the latter showed itself more clearly; the throat-affection increased; the parotid and sub-maxillary glands became much swollen; and, the disease extending finally to the larynx, the child died asphyxiated.

In September 1858, the disease appeared in Barton; and so intimately was it connected with scarlatina, that it seemed almost impossible to separate them. I was, indeed, led to believe that they were identical, and only two forms of the same disease, modified by circumstances. It was only after having carefully observed the numerous cases that came under my notice, that I became convinced that they were two distinct diseases; for, although in the commencement of the epidemic they seemed so intimately associated as to baffle all attempts to separate them, after a few weeks diphtheria began unequivocally to show itself as a distinct disease, appearing in some who had had scarlatina, and were apparently perfectly recovered; and in others who were grown up, and remembered having had scarlatina in childhood.

It is not in my power to give any proximate idea of the number of cases; the only thing possible is to give the number of deaths; and with this, I have been furnished by my friend Mr. Morley, the district registrar; and it includes all the deaths registered of scarlatina, diphtheria, ulcerated throat, etc. Nor do I know of any better plan; as though in most cases diphtheria succeeded scarlatina, there is no doubt that many of the cases registered of malignant scarlatina were affected with diphtheria at the sarne time, and, had they lived a few days longer, would probably have shown the latter disease in its characteristic form.

The number of deaths furnished by the registrar are as follows:-

\begin{tabular}{|c|c|c|c|c|c|}
\hline 1858. & rton. & & abou & & 'Total \\
\hline September & 1 & .. & 0 & .. & 1 \\
\hline October & 5) & .. & l & .. & 6 \\
\hline November & 8 & .. & 0 & .. & 8 \\
\hline December & 25 & .. & 1 & .. & 26 \\
\hline 1859. & & & & & \\
\hline January & 11 & .. & 4 & .. & 15 \\
\hline February & 2 & .. & 3 & .. & 5 \\
\hline March & 5 & .. & 7 & .. & 12 \\
\hline April & 3 & .. & 4 & .. & 7 \\
\hline May ' & 1 & .. & 5 & .. & (i \\
\hline June & 3 & . & 3 & .. & (i \\
\hline July & 3 & .. & 5 & .. & 8 \\
\hline August & 1 & .. & 0 & .. & 1 \\
\hline September & - & .. & 1 & .. & 1 \\
\hline Octuber & 0 & .. & 0 & -. & 0 \\
\hline November & 2 & .. & \pm & .. & 6 \\
\hline December & 1 & .. & 1 & .. & 2 \\
\hline 1860. & & & & & \\
\hline January & $\longrightarrow$ & . & 2 & .. & 2 \\
\hline February & 1 & .. & - & .. & I \\
\hline July & 0 & . & 1 & - & 1 \\
\hline & 72 & & 42 & & 114 \\
\hline
\end{tabular}

Of the above 114, the ages were as under; viz.: Under 1 year

$\begin{array}{rrrrr}1 & & & \\ 1 & \text { year and } & & \\ 2 & " & 3 & . . & 12 \\ 3 & " & 4 & . . & 19 \\ 4 & " & 5 & . . & 13 \\ 5 & " & 6 & . & 7 \\ 6 & \# & 7 & . . & 7 \\ 7 & " & 8 & . . & 5 \\ 8 & " & 9 & . & 6 \\ 9 & " & 10 & . & 3 \\ 10 & " & 11 & . . & 4 \\ 11 & " & 12 & . . & 4\end{array}$

